\title{
PAPER
}

\section{Association of neprilysin polymorphism with cerebral amyloid angiopathy}

\section{Yamada, N Sodeyama, Y Itoh, A Takahashi, E Otomo, M Matsushita, H Mizusawa}

See end of article for
authors' affiliations
$\ldots \ldots \ldots \ldots \ldots \ldots \ldots \ldots \ldots \ldots$
Correspondence to:
Professor M Yamada,
Department of Neurology
and Neurobiology of
Aging, Kanazawa
University Graduate School
of Medical Science, 13-1,
Takara-machi, Kanazawa
920-8640, Japan;
m-yamada@
med.kanazawa-u.ac.jp
Received
13 November 2002
Accepted in revised form
25 January 2003

\begin{abstract}
Objectives: The risk of sporadic cerebral amyloid angiopathy (CAA) may be associated with genetic polymorphisms of molecules related to anabolism or catabolism of amyloid $\beta$ protein $(A \beta)$. The authors investigated whether a polymorphism of the gene (NEP) coding for neprilysin, an enzyme catabolising $A \beta$, is associated with CAA.

Methods: The study analysed the GT repeat polymorphism in the enhancer/promoter region of NEP and severity of CAA in 164 necropsied elderly Japanese subjects.

Results: The subjects had NEP polymorphisms with 19 to 23 GT repeats and were classified into nine genotypes. CAA severity was significantly higher in the subjects with up to 40 repeats in total than those with more than 40 repeats $(p=0.005)$. There was a significant correlation between the number of the shorter alleles (19 or 20 repeats) and CAA severity $(p=0.024)$. In addition, there was no interaction between the NEP polymorphism and apolipoprotein $\mathrm{E}$ genotype.

Conclusions: These results suggest the association between the NEP polymorphism and the risk of CAA. Further study using more samples from populations with different ethnic backgrounds is necessary.
\end{abstract}

C rebral amyloid angiopathy (CAA) is a cerebrovascular amyloid deposition and causes intracerebral haemorrhage and other cerebrovascular disorders (see reviews $^{12}$ ). Several types of CAA have been identified in association with various amyloid proteins including amyloid $\beta$ protein $(A \beta)$, cystatin $C$, prion protein, transthyretin, gelsolin, and Abri/ADan. ${ }^{2}$ Sporadic CAA of A $\beta$ type is most common in elderly people as well as patients with Alzheimer's disease $(\mathrm{AD})^{2}$; polymorphisms in apolipoprotein $\mathrm{E}(A P O E)$ and other genes $^{3-7}$ may be associated with CAA or CAA related haemorrhage.

Although the pathomechanism underlying cerebrovascular amyloid deposition remains unclear, recent studies have shown that a neuronal source of $A \beta$ is sufficient to induce cerebrovascular amyloid deposition. ${ }^{8} \mathrm{~A} \beta$ in the brain extracellular fluid may be internalised by cerebrovascular smooth muscle cells, leading to vascular $A \beta$ deposition ${ }^{9}{ }^{10}$; a major molecular species of cerebrovascular $A \beta$ is the 40 -amino acid $A \beta(A \beta 40)$, although $A \beta 42$ is initially deposited in the vessel wall. ${ }^{11}$

Neprilysin has been shown to be a major proteolytic enzyme responsible for the catabolism of $A \beta$ in the brain. ${ }^{12-14} A \beta 42$ was deposited in the brain after infusion of the inhibitor, although $\mathrm{A} \beta 40$ was less affected. ${ }^{12}$ The levels of $A \beta 40$ as well as $A \beta 42$ were significantly increased in the neprilysin deficient mice in a gene dose dependent manner. ${ }^{14}$ The regional levels of $A \beta$ in the neprilysin deficient mice correlated with the vulnerability to $\mathrm{A} \beta$ deposition in the human $\mathrm{AD}$ brain..$^{14}$ In addition, localisation of neprilysin in the human cerebral cortex was inversely correlated with the vulnerability to $A \beta$ deposition ${ }^{15}$; neprilysin was reduced in high $\mathrm{A} \beta$ plaque areas of $\mathrm{AD}$ brain. ${ }^{16}$ These findings suggest an important role of neprilysin in $A \beta$ deposition.

Expression of the neprilysin gene (NEP) is transcriptionally regulated in a tissue specific manner, generating four types of neprilysin mRNA; neurons predominantly express the type 1 transcript containing exon $1 .{ }^{14-20}$ A dinucleotide GT (or TG) repeat polymorphism is present in the enhancer and promoter regions upstream of exon 1, and it may be involved in regulation of the expression level of neprilysin in neurons. ${ }^{14}{ }^{17-20}$ Therefore, the polymorphism may influence $A \beta$ degradation in the brain.
We previously examined the relation between the NEP polymorphism and $\mathrm{AD}$, but failed to find any association between them. ${ }^{21}$ As mentioned above, the major $A \beta$ species of cerebrovascular amyloid is $A \beta 40$, and this is in contrast with senile plaques in which $\mathrm{A} \beta 42$ is the most important component. Pathomechanism(s) underlying the $A \beta$ deposition would be different between cerebrovascular and brain parenchymal tissues. Therefore, we investigated whether the $N E P$ polymorphism is associated with CAA in this study. In addition, $\mathrm{AD}$ and $A P O E$ genotype were also analysed to explain the association between the NEP polymorphism and CAA.

\section{METHODS}

\section{Subjects}

We studied 164 Japanese patients (age, 62 to 104 years; mean (SD), 85.5 (7.8) years), from a consecutive necropsy series in a large geriatric hospital, excluding cases in which brain samples were not available for this study and cases of neurodegenerative diseases other than AD. We obtained consent from the families of all participants at necropsy. This study project was approved by the ethics committees of the institutions. They included 75 patients with $\mathrm{AD}$ satisfying the neuropathological criteria of the Consortium to Establish a Registry for $\mathrm{AD}$ (CERAD). ${ }^{22}$ Among the 89 non-AD patients, 32 patients clinically presented with cerebrovascular disorders, including 26 patients with cerebral infarction, four with cerebral haemorrhage, and two with both infarction and haemorrhage. There was no significant difference in the age at death between $\mathrm{AD}$ and non-AD groups. No familial cases of $\mathrm{AD}$ or CAA were included. The relation between $\mathrm{AD}$ and NEP polymorphism has already been reported in these patients. ${ }^{21}$ Some of these patients were previously studied for CAA. ${ }^{6723}$

Abbreviations: $C A A$, cerebral amyloid angiopathy; $A \beta$, amyloid $\beta$ protein; AD, Alzheimer's disease 
Table 1 Average CAA counts (mean (SE)) in the NEP genotypes

\begin{tabular}{lll}
\hline NEP genotype & CAA count & \\
\hline $19 / 19$ & $19.0(14.1)$ & $(n=4)$ \\
$19 / 20$ & $31.7(11.8)$ & $(n=10)$ \\
$19 / 21$ & $38.5(21.7)$ & $(n=4)$ \\
$20 / 20$ & $20.1(2.8)$ & $(n=102)$ \\
$20 / 21$ & $6.6(2.9)$ & $(n=23)$ \\
$20 / 22$ & $0(0)$ & $(n=5)$ \\
$20 / 23$ & $0(0)$ & $(n=2)$ \\
$21 / 21$ & $18.0(9.2)$ & $(n=11)$ \\
$21 / 23$ & $3.7(3.7)$ & $(n=3)$ \\
& $18.0(2.1)$ & $(n=164)$ \\
Total & & \\
\hline
\end{tabular}

$\mathrm{p}=0.077$ by Kruskal-Wallis test.

\section{Neuropathological evaluation of CAA}

Congophilic deposits with green birefringence under polarised light were identified as amyloid deposits. The cerebrovascular amyloid deposits were immunohistochemically confirmed to be $A \beta$. Using a large section of the occipital lobe (about $4 \times 4 \mathrm{~cm}$ in size), the numbers of meningeal and cortical vessels with and without amyloid deposits were counted, and the percentage of amyloid laden vessels was calculated (= CAA count). We examined the occipital lobe, because it was the most frequently affected by CAA in our previous study in both $\mathrm{AD}$ and non-AD cases ${ }^{24}$ and seems suitable to detect CAA of very slight degree. The quantification was performed without knowledge of the NEP or APOE genotypes. Severe vascular wall involvement by CAA was commonly found in patients with high CAA counts.

\section{Identification of the NEP polymorphism}

The NEP polymorphism was identified as previously reported. ${ }^{21}$ Briefly, genomic DNA, isolated from frozen brain tissue, was amplified by PCR as described by Comings et al. ${ }^{25}$ GT repeats in the 5' region of NEP were counted with an ALF DNA sequencer II (Pharmacia Biotech). Direct sequence analysis of PCR products of some patients with representative genotypes using ABI PRISM model 310 (Perkin-Elmer) verified the number of GT repeats. The APOE genotype was also determined. ${ }^{21}$

\section{Statistical analyses}

We compared the number of CAA counts among the NEP genotypes. We also compared the total repeat number and other parameters of the NEP polymorphism. Similar analyses were performed according to the subgroups $\mathrm{AD}$ or non-AD, and $A P O E \in 4$ status. Kruskal-Wallis, Mann-Whitney, and Spearman's rank correlation tests were used. Statistical significance was defined as $\mathrm{p}<0.05$. The statistical analyses were performed using StatView J-7.5 (Abacus Concepts, Berkeley, CA).

\section{RESULTS}

The subjects had NEP polymorphisms with 19 to 23 GT repeats and were classified into nine genotypes (table 1). The allele with 20 repeats was the most frequent (allele frequency, 0.74 ), followed by $21(0.16), 19(0.07), 22(0.02)$, and 23 repeats (0.02). There was no difference in the NEP allele frequency between $\mathrm{AD}$ and non-AD cases as previously reported. ${ }^{21}$

Among the 164 patients, 93 presented with CAA of various degrees, and 71 had no CAA. CAA counts were not significantly different between the NEP genotypes in total (table 1), AD, or non-AD cases (data not shown). As shorter alleles tended to be associated with a higher CAA count (table 1 ), we examined the relation between the total repeat number and CAA. As shown in table 2, patients with up to 40 repeats in total had a significantly higher CAA count $(21.6(2.7))$ than those with more than 40 repeats $(8.2(2.8))(\mathrm{p}=0.005)$; the difference was also significant in non- $\epsilon 4$ carriers, but not in $\mathrm{AD}$, non- $\mathrm{AD}$, or $A P O E \in 4$ carriers.

Furthermore, the number of the shorter (19 or 20) repeat alleles significantly correlated with CAA count in the total cases $(9.9$ (7.9) for no alleles; 9.0 (3.5) for one allele; 21.6 (2.7) for two alleles) (correlation coefficient $=0.176$ and $\mathrm{p}=0.024$ by Spearman's rank correlation test), but not in the subgroups by $\mathrm{AD}$ or non- $\mathrm{AD}$, or in those by $A P O E \in 4$ carriers or non- $\epsilon 4$ carriers (data not shown).

In addition, the presence of $A P O E \in 4$ was significantly associated with $\mathrm{AD}$ in this population (frequency of $\epsilon 4$ carriers: 0.45 in $\mathrm{AD}$, and 0.15 in non- $\mathrm{AD})(\mathrm{p}<0.0001)$. AD patients had a significantly higher CAA count $(30.3(3.5))$ than non-AD patients $(7.7(2.1)) \quad(\mathrm{p}<0.0001)$. APOE $\in 4$ carriers had a significantly higher CAA count (23.2 (3.9)) than non- $\epsilon 4$ carriers $(15.9(2.5))(p=0.0058)$, but this association was not significant within the $\mathrm{AD}$ or non-AD groups. There was no association between the NEP polymorphism (the total repeat number or the number of shorter alleles) and $A P O E$ genotype (data not shown).

\section{DISCUSSION}

We showed that the CAA severity was significantly higher in the patients who had an NEP polymorphism with up to 40 GT repeats in total than in those with more than 40 repeats. The shorter allele with 19 or 20 repeats was associated with a higher CAA severity.

As the NEP polymorphism was not associated with $\mathrm{AD}$ in this population, ${ }^{21}$ the association between the NEP polymorphism and CAA cannot be explained by an association with $\mathrm{AD}$, a strong risk factor of CAA. The association between the $N E P$ polymorphism and CAA did not seem to be influenced by the presence of $\mathrm{AD}$ or by the status of $A P O E \in 4$. There was no interaction between the $N E P$ polymorphism and $A P O E$ genotype.

In contrast with our previous study, ${ }^{21}$ Streffer et al found a positive association between the NEP polymorphism and AD ( $\mathrm{J}$ R Streffer, et al, 31st Annual Meeting of Society for Neuroscience, San Diego, 10-15 November, 2001). The 20-repeat

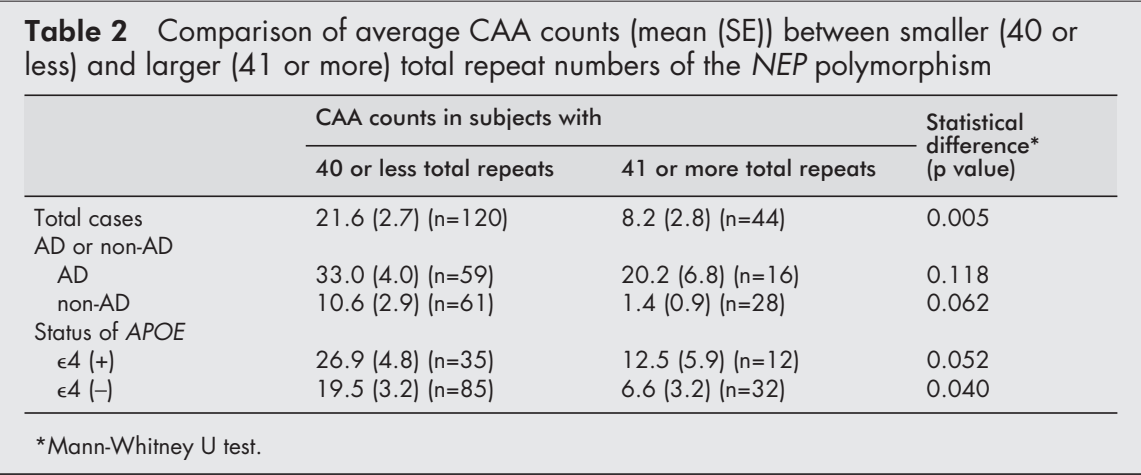


allele was the most frequent in our Japanese population, while the 21-repeat allele was the most frequent in their European population ( $\mathrm{J}$ R Streffer, et al, 31st Annual Meeting of Society for Neuroscience). There may be an ethnic difference in the NEP polymorphism. In addition, another recent study with clinical samples from a Japanese population showed lack of association between the NEP polymorphism and $\mathrm{AD}{ }^{26}$

As the NEP polymorphism is present in the regulatory region upstream of exon 1 , this polymorphism may influence the transcription of NEP to type 1 mRNA in neurons ${ }^{17-20}$; the dinucleotide repeats may induce conformational changes in the DNA helix that results in changed DNA-protein interactions. ${ }^{19}$ The shorter alleles may be associated with decreased levels of the neuronal NEP mRNA transcripts, which would result in a decrease of neprilysin activity. In such cases, the decreased neprilysin activity would increase the levels of both $A \beta 40$ and $A \beta 42$ as suggested in the experiment with neprilysin deficient mice. ${ }^{14}$ The increased $A \beta 40$ and $A \beta 42$ levels would be linked to a risk of CAA, but the reason why the $N E P$ polymorphism was not associated with senile plaques ${ }^{21}$ is unknown.

In addition, Comings et $a^{25}$ reported that low molecular weight alleles for this repeat polymorphism are associated with a low amplitude of P300, a cognitive event related brain potential. They suggested that the lower molecular weight alleles-that is, shorter alleles, are associated with increased levels of enkephalinase (neprilysin) and thus lower CNS enkephalin levels. ${ }^{25}$ Their presumption is not consistent with ours concerning $\mathrm{A} \beta$ catabolism. It is possible that the association between NEP polymorphism with CAA reflects linkage disequlibrium with another polymorphism in NEP or a nearby gene. The significance of this polymorphism in the expression of $N E P$ and neprilysin activity for proteolysis of $\mathrm{A} \beta$ and roles of other NEP polymorphisms should be further clarified.

Finally, this is the first report suggesting that a genetic polymorphism of an $\mathrm{A} \beta$ degradating enzyme may be associated with risk of CAA. However, this study is limited to a comparatively small sample population obtained from an necropsy series in a geriatric hospital. Our results warrant further study with a larger sample size from populations with various ethnic backgrounds. Although the significance and biological background of this association need to be established, this polymorphism is expected to become a useful biological marker for clinical diagnosis of CAA, and modulation of neprilysin activity would be a therapeutic target.

\section{Authors' affiliations}

M Yamada, Department of Neurology and Neurobiology of Aging, Kanazawa University Graduate School of Medical Science, Kanazawa, Japan

N Sodeyama, H Mizusawa, Department of Neurology and Neurological Science, Tokyo Medical and Dental University, Tokyo, Japan

Y Itoh, E Otomo, Department of Internal Medicine, Yokufukai Geriatric Hospital, Tokyo, Japan

A Takahashi, Department of Organ and Function Pathology, Yokufukai Geriatric Hospital, Tokyo, Japan

M Matsushita, Department of Neuropathology, Tokyo Institute of Psychiatry, Tokyo, Japan

Funding: the study was supported by grants for the Amyloidosis Research Committee (to MY) and the Research Committee of Genetic Analyses of Cerebrovascular Disorders (to MY) from the Ministry of Health, Labour and Welfare, Japan, and by a Grant-in-Aid for Scientific Research (to
MY) from the Ministry of Education, Culture, Sports, Science and Technology, Japan.

Competing interests: none declared.

\section{REFERENCES}

1 Vinters HV. Cerebral amyloid angiopathy: a critical review. Stroke 1987; 18:311-24

2 Yamada $M$. Cerebral amyloid angiopathy: an overview. Neuropathology 2000;20:8-22.

3 Greenberg SG, Rebeck GW, Vonsattel JPG, et al. Apolipoprotein E $\in 4$ and cerebral hemorrhage associated with amyloid angiopathy. Ann Neurol 1995:38:254-9.

4 Premkumar DRD, Cohen DL, Hedera $P$, et al. Apolipoprotein $E \epsilon 4$ alleles in cerebral amyloid angiopathy and cerebrovascular pathology associated with Alzheimer's disease. Am J Pathol 1996;148:2083-95

5 Nicoll JAR, Burnett C, Love S, et al. High frequency of apolipoprotein $E$ $\epsilon 2$ in hemorrhage due to cerebral amyloid angiopathy. Ann Neurol 1997:41:716-21.

6 Yamada M, Sodeyama N, Itoh Y, et al. Association of presenilin-1 polymorphism with cerebral amyloid angiopathy in the elderly. Stroke 1997;28:2219-21

7 Yamada M, Sodeyama N, Itoh Y, et al. Association of $\alpha$ l-antichymotrypsin polymorphism with cerebral amyloid angiopathy. Ann Neurol 1998:44:129-31.

8 Calhoun ME, Burgermeister P, Phinney AL, et al. Neuronal overexpression of mutant amyloid precursor protein results in prominent deposition of cerebrovascular amyloid. Proc Natl Acad Sci USA 1999;96: 14088-93.

9 Urmoneit B, Prikulis I, Wihl G, et al. Cerebrovascular smooth muscle cells internalize Alzheimer amyloid beta protein via a lipoprotein pathway: implications for cerebral amyloid angiopathy. Lab Invest 1997;77:157-66

10 Weller RO, Massey A, Newman TA, et al. Cerebral amyloid angiopathy. Amyloid $\beta$ accumulates in putative interstitial fluid drainage pathways in Alzheimer's disease. Am J Pathol 1998;153:725-33.

11 Shinkai Y, Yoshimura M, Ito Y, et al. Amyloid $\beta$-proteins $1-40$ and $1-42(43)$ in the soluble fraction of extra- and intracranial blood vessels. Ann Neurol 1995;38:421-8

12 Iwata N, Tsubuki S, Takaki Y, et al. Identification of the major A 31 -42-degrading catabolic pathway in brain parenchyma: suppression leads to biochemical and pathological deposition. Nat Med 2000; 6: 143-50.

13 Takaki Y, Iwata N, Tsubuki S, et al. Biochemical identification of the neutral endopeptidase family member responsible for the catabolism of amyloid $\beta$ peptide in the brain. J Biochem 2000;1 28:897-902.

14 Iwata N, Tsubuki S, Takaki Y, et al. Metabolic regulation of brain A $\beta$ by neprilysin. Science 2001;292:1550-2.

15 Akiyama H, Kondo H, Ikeda K, et al. Immunohistochemical localization of neprilysin in the human cerebral cortex: inverse association with vulnerability to amyloid $\beta$-protein $(A \beta)$ deposition. Brain Res 2001;902:277-81.

16 Yasojima K, Akiyama H, McGeer EG, et al. Reduced neprilysin in high plaque areas of Alzheimer brain: a possible relationship to deficient degradation of $\beta$-amyloid peptide. Neurosci Lett 2001;297:97-100.

17 Li C, Mooze RM, Hershi LB. Tissue-specific expression of rat neutral endopeptidase (neprilysin) mRNAs. J Biol Chem 1995;270:5723-8.

18 Haouas H, Morello D, Lavenu A, et al. Characterization of the $5^{\prime}$ region of the CD 10/neutral endopeptidase 24.11 gene. Biochem Biophys Res Commun 1995;207:933-42.

19 Ishimaru F, Shipp MA. Analysis of the human CD10/neutal endopeptidase 24.11 promoter region: two separate regulatory elements. Blood 1995:85:3199-207.

20 Li C, Hersh LB. Characterization of the promoter region of the rat neprilysin gene. Arch Biochem Biophys 1998;358:189-95.

21 Sodeyama N, Yamada M, Itoh Y, et al. Lack of association of neprilysin polymorphism with $A D$ and $A D$ type neuropathological changes. $J$ Neurol Neurosurg Psychiatry 2001;71:817-18.

22 Mirra S, Heyman A, McKeel D, et al. The Consortium to Establish a Registry for Alzheimer's Disease (CERAD), Part II: standardization of the neuropathologic assessment of Alzheimer's disease. Neurology 1991:41:479-86.

23 Yamada M, Sodeyama N, Itoh Y, et al. No association of paraoxonase genotype or atherosclerosis with cerebral amyloid angiopathy. Stroke 2002;33:896-900.

24 Yamada $M$, Tsukagoshi $H$, Otomo $E$, et al. Cerebral amyloid angiopathy in the aged. J Neurol 1987;234:371-6.

25 Comings DE, Dietz G, Johnson JP, et al. Association of the enkephalinase gene with low amplitude $\mathrm{P} 300$ waves. NeuroReport 1999:10:2283-5.

26 Oda M, Morino H, Maruyama H, et al. Dinucleotide repeat polymorphisms in the neprilysin gene are not associated with sporadic Alzheimer's disease. Neurosci Lett 2002;320:105-7. 